\title{
Riccardo Giacconi (1931-2018)
}

\author{
Astronomer who opened the $\mathrm{X}$-ray window onto the Universe.
}

$\mathrm{R}$ evolutionary discoveries in astronomy stem from new observing technologies. And so it was with orbiting $\mathrm{X}$-ray telescopes. They revealed a Universe dominated by luminous, gravity-fed black holes and plasmas glowing at tens of millions of degrees, inside clusters of galaxies. Now 60 years old, the discipline requires space observatories - outside Earth's X-rayblocking atmosphere - and new, specialized optics and detectors. For pioneering these, Riccardo Giacconi won the 2002 Nobel Prize in Physics.

Giacconi shaped the tools and culture of modern astronomy, which centres on large facilities that serve the community. He realized the importance of catering to the entire observatory life cycle, across the electromagnetic spectrum - from conception to hardware implementation and operations, including calibrations, software pipelines, data archives and software tools for users. $\mathrm{He}$ championed scientific leadership and management, and integrated teams, with members doing both technical work and research. His approach is now integral to all major international observatories. One result is the adoption of data interoperability standards for astronomy, developed by the International Virtual Observatory Alliance since 2002.

Giacconi died on 9 December 2018 in San Diego, California, aged 87. He was born in Genoa, Italy, in 1931. His mother, who taught in a secondary school and wrote mathematics textbooks, spurred him to excel. In 1956, with a doctorate on cosmic-ray detection from the University of Milan, he moved to the United States, as a Fulbright fellow at Indiana University Bloomington and later became a research associate at Princeton University in New Jersey. In 1959, Giacconi joined the Massachusetts Institute of Technology (MIT) spin-off company American Science \& Engineering (AS\&E), then in Cambridge.

Here, with Bruno Rossi of MIT and other AS\&E physicists, Giacconi conducted a series of experiments on the detection of X-rays from space. In 1962, with Geiger counters flown on an Aerobee rocket from the US White Sands Missile Range in New Mexico, Giacconi and his collaborators discovered the first celestial X-ray source (Sco X-1). It was later identified as a neutron star that was accreting matter from a lowmass star companion. The team also detected a pervasive celestial X-ray background.

These discoveries spurred Giacconi's proposal to NASA for a strategic programme

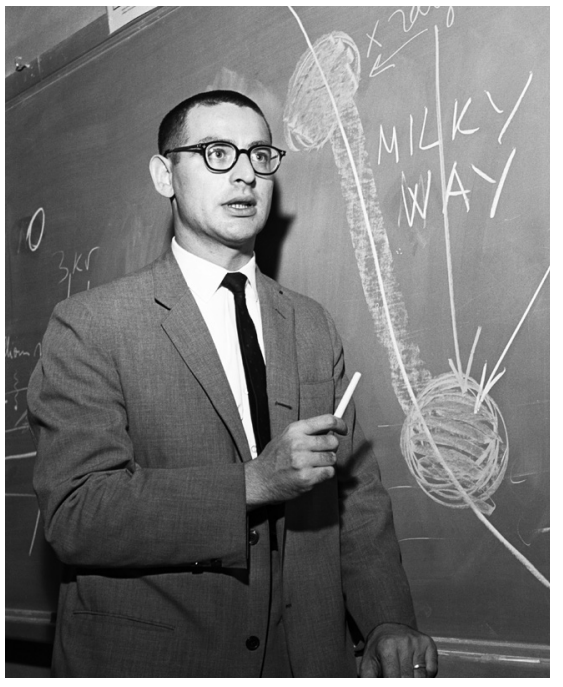

of X-ray observatories. The first, NASA's Small Astronomy Satellite 1, was launched from the Italian San Marco platform off the coast of Kenya, on 12 December 1970 - Kenya's independence day - and was renamed Uhuru, Swahili for 'freedom'. Uhuru performed the first X-ray survey of the entire sky. It led to the discovery of accreting neutron-star and black-hole sources in binary stars, X-ray emission from supernova remnants, active galactic nuclei and hot haloes in clusters.

In 1973, Giacconi and his group moved to the Harvard-Smithsonian Center for Astrophysics. Here, he led the collaboration that built and operated the first imaging $\mathrm{X}$-ray telescope, the Einstein Observatory. This detected X-rays from almost all types of object in the cosmos, establishing the technique as a branch of astronomy.

Understanding the X-ray background drove Giacconi. It led him to pursue telescopes with increasingly higher image resolution - notably, the Chandra X-ray Observatory, NASA's flagship mission launched on the Space Shuttle Columbia in July 1999. Thanks to Chandra, we know that this background is mostly due to giant black holes with masses up to one billion times that of the Sun, swallowing gas clouds and stars.

Early X-ray observations presented a whole new set of phenomena. They showed that, in some cases, the energy produced by celestial bodies is more than can be observed in visual light. These findings underpin the present understanding of the evolution of binary stars, galaxies and the giant black holes at their centres, and of the Universe at large.
Giacconi led the opening up of space observatories to general use - not just by specialist operating teams - and the establishment of calibrated data archives. Such archives are now widely implemented by NASA, and are gaining ground in the new generation of large, ground-based observatories. As a result, more astronomers are embracing multi-wavelength observations, from radio to X-rays. Archival data get re-used, increasing their discovery potential with time (a typical Chandra data set is re-published about six times). This openness has catalysed a better understanding of the Universe and its evolution.

In 1981, Giacconi became the first director of the Space Telescope Science Institute (STScI) at the Johns Hopkins University in Baltimore, Maryland, which operates NASA's Hubble Space Telescope. His management style, developed during his AS\&E years, shaped the STSCI into one of the premier astronomy centres worldwide. He took the same tack when he became directorgeneral of the European Southern Observatory (ESO) in Garching, Germany, in 1992. At the ESO, he oversaw the development of the highly successful Very Large Telescope in Chile, and was involved in the early development of the revolutionary Atacama Large Millimeter/submillimeter Array (ALMA). He continued to oversee ALMA, together with other major US radio telescopes, during his five-year tenure as president of Associated Universities, Inc., which started in 1999.

Science was Giacconi's driving force. His professional, social and family lives were often intertwined, with friends and engagements worldwide. Architecture and art were important to him: he enjoyed copying Renaissance paintings, with which he decorated his home.

He could be blunt and push controversial ideas, but he appreciated a vigorous discussion. I remember his disappointment during the Harvard years that people did not argue with him any more. As Giacconi wrote in his autobiography, Secrets of the Hoary Deep (2008), he needed to explore uncharted seas. He opened new realms to discovery.

\section{Giuseppina Fabbiano is senior} astrophysicist at the Center for Astrophysics | Harvard \& Smithsonian, and head of the Chandra X-ray Center Data Systems Division. She met Giacconi in the early 1970s, while working on Uhuru data for her doctoral thesis. In 1975, she joined his High Energy Division of the Center for Astrophysics. e-mail:gfabbiano@cfa.harvard.edu 